\title{
Probabilistic: A Fuzzy Logic-Based Distance Broadcasting Scheme For Mobile Ad Hoc Networks
}

\author{
Tasneem Bano \\ Computer Science and Engineering, \\ Maulana Azad National Institute of Technology \\ Bhopal, Madhya Pradesh 462051, India
}

\author{
Jyoti Singhai \\ Electronics and Engineering, \\ Maulana Azad National Institute of technology \\ Bhopal, Madhya Pradesh 462051, India
}

\begin{abstract}
An on-demand route discovery method in mobile ad hoc networks (MANET) uses simple flooding method, whereas a mobile node blindly rebroadcasts received route request (RREQ) packets until a route to a particular destination is established. Thus, this leads to broadcast storm problem. This paper presents a novel algorithm for broadcasting scheme in wireless ad hoc networks using a fuzzy logic system at each node to determine its capability to broadcast route request packets, based on the node location. Our simulation analysis shows a significant improvement in performance in terms of routing overhead, MAC collisions and end-to-end delay while still achieving a good throughput compared to the traditional AODV.
\end{abstract}

Keywords Broadcasting; Distance based broadcasting; Fuzzy; Optimization Technique; AODV.

\section{INTRODUCTION}

Traditionally, broadcasting means sending a message from one given node (the source station) to all the nodes in the network. In a (multi-hop) decentralized network, the broadcasted data has to be relayed by intermediate nodes in such a way that the entire network graph is spanned. In MANET, simplistic broadcast schemes result in network with redundancy, contention, and collision which is often called 'Broadcast Storm Problem'. This can prevent broadcasts from achieving the objectives of optimal delivery ratio, energy balancing, and latency.

The main objective of a broadcasting scheme is to avoid broadcast storm problems and to provide good network performance and scalability. Therefore, a route discovery technique that can guarantee an efficient utilization of the limited system resources while achieving acceptable levels of other important performance metrics such as throughput and end-to-end delay is highly desirable. Till date, research on efficient broadcasting schemes in mobile ad-hoc networks has proceeded along two main schemes:

\section{A. Deterministic Schemes}

\section{B. Probabilistic Schemes}

Deterministic Schemes use network topological information to build a virtual backbone that covers all the nodes in the network. In order to build a virtual backbone, nodes exchange information, typically about their immediate or two hop neighbors. This results in a large overhead in terms of time and message complexity for building and maintaining the backbone, especially in the presence of mobility.
Probabilistic Schemes, however, rebuild a backbone from scratch during each broadcast. Nodes make instantaneous local decisions about whether to broadcast a message or not using information derived only from overheard broadcast messages. These schemes incur a smaller overhead and demonstrate superior adaptability in changing environments when compared to deterministic schemes [1, 2, 4, 17].

The rest of the paper is organized as follows. Section II presents related work on some route discovery techniques. Section III presents analysis of node location, while section IV gives an introduction of fuzzy logic based distance route discovery method. Section V and VI gives simulation parameter, conducts a comparison and performance evaluation of the proposed route discovery methods. Finally, Section VII concludes the study and scope of future research work.

\section{RELATED WORK}

One of the earliest broadcast mechanisms in both wired and wireless networks is flooding, where every node in the network retransmits a message to its neighbors upon receiving it for the first time. Although flooding is simple and easy to implement, it can be costly in terms of network performance, and may lead to a serious problem, often known as the broadcast storm problem $[4,5,7]$. The broadcast problem is then characterized by high redundant message retransmissions, network bandwidth contention, and collision. $\mathrm{Ni}$ [4] have studied the flooding protocol and the results obtained have shown that rebroadcast could provide at most $61 \%$ additional coverage and only $41 \%$ additional coverage in average over that already covered by the previous broadcast. As a result, they have concluded that rebroadcasts are very costly and should be used with caution.

Probabilistic broadcasting is one of the simplest and most efficient broadcast techniques that have been suggested [6] in the literature. The advantage of probabilistic broadcasting over the other proposed broadcast methods [6, 12 and 13] is its simplicity. However, studies $[6,11]$ have shown that although probabilistic broadcast schemes can significantly reduce the degrading effects of the broadcast storm problem [6], they suffer from poor reachability, especially in a sparse network topology. In this approach each intermediate node rebroadcasts received packets only with a predetermined forwarding probability. Clearly, the appropriate choice of the forwarding probability determines the effectiveness of this technique. 
Most probabilistic broadcast approaches that have been proposed in the literature [6,8 and 11] have considered a fixed forwarding probability at each intermediate node. This could lead to most nodes not receiving the broadcast packet when the forwarding probability is set too low or more redundant transmissions if the probability is set too high, as discussed in $[9,10]$. One of the causes for this stems from the fact that every node in the network has the same probability of rebroadcast, regardless of its local topological characteristics, such as neighboring node location. In a dense network multiple nodes may share similar transmission coverage. Therefore, if some nodes, randomly, do not forward the broadcast packet, these could save resources without degrading the delivery effectiveness. On the other hand, in a sparse network, there is much less shared coverage; thus some nodes might not receive the broadcast packet unless the rebroadcast probability is set high enough. Consequently, the rebroadcast probability should be set differently from one node to another according to their local topological characteristics.

The author in [15] introduces the concept of distance into the counter based scheme, in which nodes closer to the border of source has been given higher rebroadcast probability since they create better expected additional coverage area [6]. Distance threshold is taken to distinguish between interior and border nodes. Two distinct random assessment delays are applied to the border and interior nodes, with border nodes having shorter random assessment delay than the interior nodes.

This paper proposes a new route discovery algorithm that utilizes probabilistic broadcast methods using fuzzy logic to disseminate the RREQ packets. To evaluate the new route discovery methods we have considered using the AODV [14] routing algorithm and have compared the performance of fuzzy logic based broadcasting with the Distance-Aware Counter-Based Broadcast Scheme [15]. Our results reveal that equipping AODV [14] with the new fuzzy logic based probabilistic route discovery methods help to reduce the overall routing overhead while achieving good network throughput with improved end-to-end delay when compared to the traditional AODV [14] and [15].

\section{Analysis Of Node Location}

In Mobile ad hoc networks node location is one of the most important aspects in broadcasting. Each node has its transmission range within which its neighbor can receive broadcasting information [16]. Consider a simple scenario in figure below

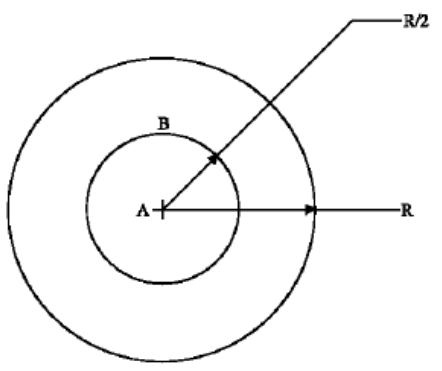

Figure 1. Coverage area of node A and B
Host a sends a broadcast message and host $\mathrm{B}$ decides to rebroadcast the message. Let $C_{A}$ and $C_{B}$ denotes the circle area covered by A's and B's transmission, respectively. The additional area provided by B's rebroadcast is denoted by $C_{B-A}$. Let $\mathrm{r}$ be the radii of $C_{A}$ and $C_{B}$ and $\mathrm{d}$ the distance between A and B. than $\left|C_{B-A}\right|=\left|C_{A}\right|-\left|C_{A \cap B}\right|=\pi r^{2}$ - INTC (d), where INTC (d) is the intersection area of the two circles centered at 2 points discussed by d:-

$\operatorname{INTC}(\mathrm{d})=4 \int_{d / 2}^{r} \sqrt{r^{2}+r^{2}} \mathrm{dx}$

When $\mathrm{d}=\mathrm{r}$, the coverage area $\left|C_{B-A}\right|$ is the largest which equals $\pi r^{2}$ - INTC $(\mathrm{d})=0.61 \pi r^{2}$. This shows that $61 \%$ of additional coverage can be provided over that already covered by the previous transmission[6].

And when $\mathrm{d}<\mathrm{r}$ that is $\mathrm{B}$ is located in As' transmission range, then the average value can be obtained by integrating the above value over the circle of radius $\mathrm{x}$ centered at $\mathrm{A}$ for $\mathrm{x}$ in $[0, \mathrm{r}]$

$\int_{0}^{r} \frac{2 \pi x\left[\pi r^{2}-\operatorname{INTC}(x)\right]}{\pi r^{2}} \mathrm{dx} \sim 0.41 \pi r^{2}$

So now a broadcast can cover only additional $41 \%$ area in average.

Now, by considering the expected additional coverage area of a node, different broadcasting probability can be set for nodes with $\mathrm{d}=\mathrm{r}$ [border nodes] and $\mathrm{d}<\mathrm{r}$ [interior nodes]. In order to distinguish these two types of node we introduce Dth [distance threshold] $=200$ when $\mathrm{R}$ [transmission radius] $=250$. To calculate $\mathrm{P}$, we need the relative distance between nodes $D_{A B}$ between nodes A and B then

$\mathrm{Pi}=\left(\frac{D_{A B}}{R}\right)^{n} \times 100$

When $n=0$ the scheme is simple flooding, if $n=1$, the scheme broadcast with $\mathrm{Pi}$ if it receives the packet for first time, otherwise discard the packet. Now when $n>1, P i$ increases exponentially, it makes retransmit nodes concentrate towards the border of source nodes coverage area, which results in the increasing of the EAC area of next hop and less rebroadcasts. The concentration increases with $\mathrm{n}$ values. The larger $\mathrm{n}$ is selected, the more concentration of retransmission nodes to the border. The value of $\mathrm{n}$ is selected based on the network densities. Now varying the value of $\mathrm{n}$ from 0,1 and $>1$ and see the effect on the redundant area.

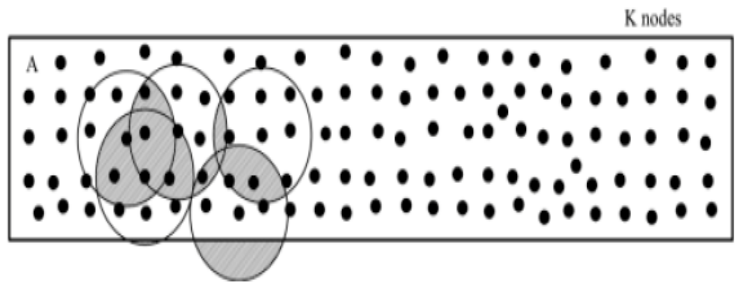

Figure 2. Overlapped coverage area

Let $\mathrm{S}$ is the area of our topology area A. there are m nodes in A and transmission radius is R. then every nodes' coverage area is $\mathrm{Si}=\pi r^{2}$. Now when $\mathrm{n}=0$

$S_{Z}{ }^{1}=\sum_{i=1}^{m} S i$ 
Then,

$S_{Z}{ }^{1}=\sum_{i=1}^{m} \pi R^{2}=\mathrm{m} \pi R^{2}$

Now in above figure there is overlapping in the coverage area as the network is dense. Then the redundant coverage area is:-

$S_{r}^{2}=S_{Z}-S$

$=\mathrm{m} \pi R^{2}-\mathrm{S}$

When $n=1$ the probability scheme broadcast with Pi

$\mathrm{Si}=\operatorname{Pi} \pi R^{2} \quad\{\mathrm{i} \leq[1, \mathrm{~m}]\}$

The total area can be calculated as:

$S_{Z}^{2}=\sum_{i=1}^{m} S i \rightarrow \sum_{i=1}^{n} P i \pi R^{2}$

$S_{Z}{ }^{2}=\sum_{i=1}^{m}\left(\frac{L i j}{R}\right) \pi R^{2} \rightarrow \sum_{i=1}^{m} L i j \pi R$

(4)

Total redundant area can be calculated as:-

$S_{r}^{2}=S_{Z}^{2}-\mathrm{S}$

$=\sum_{i=1}^{m} L i j \pi R-\mathrm{S}$

Since $\mathrm{Lij}<\mathrm{R}$, the distance between two connecting nodes will not exceed the transmission radius. So we can conclude that from equation 3 and 5

$\mathrm{m} \pi R^{2}>\sum_{i=1}^{m} \operatorname{Lij} \pi R$

That is $S_{r}{ }^{1}>S_{r}{ }^{2}$ this means the total redundant area of fixed probability is smaller than simple flooding. Similarly when $\mathrm{n}>1$, total area can be calculated

$S_{Z}^{3}=\sum_{i=1}^{m}\left(\frac{L i j}{R}\right)^{n} \pi R^{2}$

$S_{Z}^{3}=\sum_{i=1}^{m} \pi \frac{L i j^{n}}{R^{n-2}}$

As derived that from equation 4

$S_{Z}^{2}=\sum_{i=1}^{m} \pi L i j R$

Let $\quad S_{Z}^{2}=\sum_{i=1}^{m} \pi\left(\frac{L i j}{R}\right)^{n-1}\left(\frac{L i j^{n}}{R^{n-2}}\right)$

Then replace the equation with $S_{Z}{ }^{3}$ from equation 6 we get

$$
S_{Z}^{2}=\sum_{i=1}^{m} \pi\left(\frac{R}{L i j}\right)^{n-1} S_{Z}^{3}
$$

As stated Lij is smaller than $\mathrm{R}$ and $\mathrm{n}>1$ so:-

$\left(\frac{R}{L i j}\right)^{n-1}>1$

$S_{Z}{ }^{2}>S_{Z}{ }^{3}$ and $S_{r}{ }^{2}>S_{r}{ }^{3}$ can be deduced

Hence the redundant area is reduced by broadcasting with a probability value based on the node location. By considering the above analysis we conclude that border nodes should have higher probability value.

\section{FUZZY LOGIC-BASED DISTANCE BROADCASTING SCHEME}

A probabilistic route discovery approach can be developed which can further reduce the route discovery overhead by exploiting the problem solving control system methodology that is fuzzy logic. The Fuzzy Logic algorithm is illuminated by the powerful capability of fuzzy logic system to handle uncertainty and ambiguity. Fuzzy logic system is well known as model free. Their membership functions are not based on statistical distributions. In this paper, we apply fuzzy logic system to optimize the broadcasting scheme in AODV based on the node location. The main goal is designing the algorithm to use Fuzzy Logic Systems so as to avoid the broadcast storm problem.

For the inference process we use Mamdani's method. Mamdani's method is most commonly used in applications. There are four steps to get the crisp value from the FIS system.

1) The first step is to evaluate the antecedent for each rule.

2) The second step is to obtain each rule's conclusion.

3) The third step is to aggregate conclusions.

4) The fourth and last is defuzzification

The defuzzified output is then given as input into probability broadcasting of the AODV RREQ module. In this work fuzzy logic is embedded in Qualnet so as to have performance analysis of a dynamic nature.

\section{B. Fuzzy Logic Controller}

Fuzzy logic control is derived from fuzzy set theory introduced by Zadeh in 1965. The Fuzzy Logic Controller (FLC) shows a better performance than conventional controllers in the form of increased robustness. Fuzzy Control is based upon practical application knowledge represented by so called linguistic rule based, rather than by analytical (either empirical or theoretical) models. Fuzzy Control can be used when there is an expertise that can be expressed in its formalism. Other advantages of FLC are:

1) It can work with less precise inputs.

2) It does not need fast processors.

3) It needs less data storage in the form of membership functions and rules than conventional look up table for nonlinear controllers.

\section{Fuzzification}

Fuzzy logic uses linguistic variables instead of numerical variables. The process of converting a numerical variable (real number or crisp variable) into a linguistic variable (fuzzy number) is called fuzzification.

The simplest form of membership function is triangular membership function and it is used here as the reference. To determine the broadcasting probability value, one input function transforms the system inputs into fuzzy sets which is node location.

Table I below shows the membership value for the input node location and figure 3 shows the input Membership Function. 
TABLE 1: INPUT LINGUISTIC VARIABLE

\begin{tabular}{|l|lr|}
\hline Input & Membership & \\
\hline Node location & $\begin{array}{l}\text { Border, } \\
\text { border, } \\
\text { Interior }\end{array}$ & $\begin{array}{r}\text { Internal } \\
\text { Exterior, }\end{array}$ \\
\hline
\end{tabular}

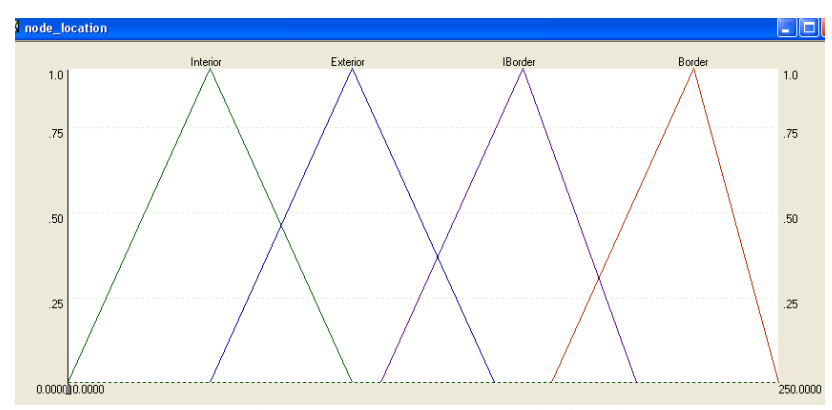

Figure 3. Input membership function

The output function is composed of four membership functions as seen in figure 4. Table II gives the output functions.

TABLE II: OUTPUT LINGUISTIC VARIABLE

\begin{tabular}{|l|l|}
\hline Output & Membership \\
\hline Probability & $\begin{array}{l}\text { High, Medium, Low, } \\
\text { very Low }\end{array}$ \\
\hline
\end{tabular}

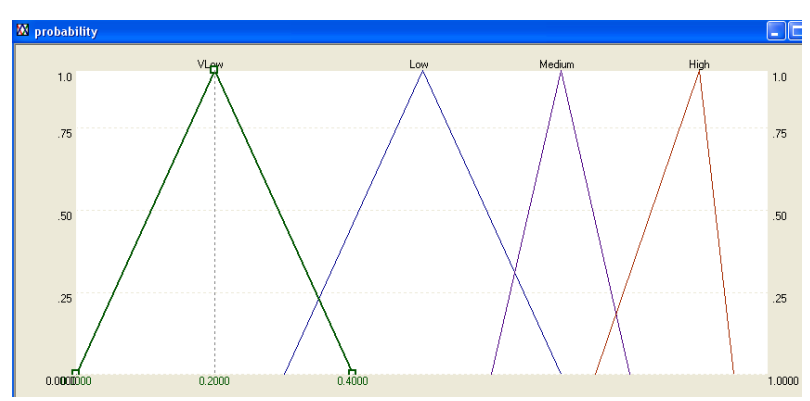

Figure 4. Output membership function

\section{Rule base table and inference engine}

The rules are in the format - ,If else. Then, the if ,part "ef a rule is called the rule-antecedent and is a description of a process state in terms of a logical combination of atomic fuzzy propositions. The ,then" part of the rule is called the rule consequent and is a description of the control output in terms of logical combinations of fuzzy propositions. In our system, we have 4 rules in the fuzzy inference. The form of the rules is: IF A, THEN C. The A and C represent node location and probability respectively.

\section{E. Defuzzification}

The reverse of fuzzification is called defuzzification. The use of FLC inference engine produces required output in a linguistic form. After aggregating the conclusions obtained by each rule, a defuzzification method is still needed to get the crisp value. One of the most popular defuzzification methods is the Centroid, which returns the center of the area under the fuzzy set obtained aggregating conclusions. The Centroid is shown in figure 5.

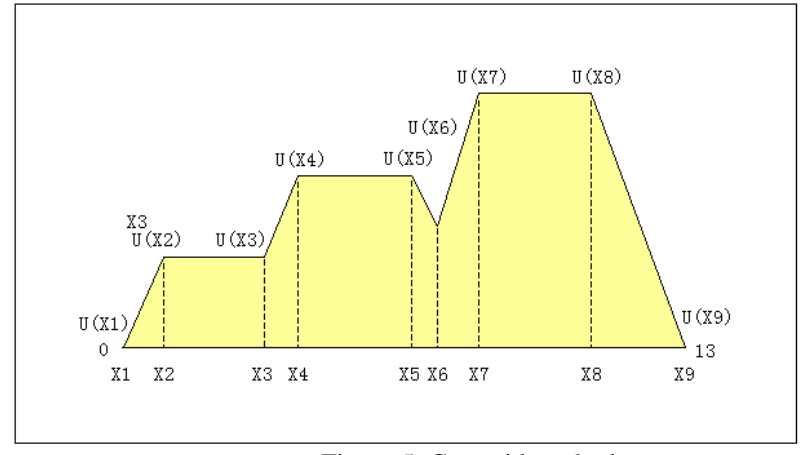

Figure 5. Centroid method

\section{SIMULATION}

Qualnet is a discrete event simulator used in the simulation of mobile ad-hoc networks. To evaluate the performance of the proposed scheme for route discovery algorithms, the implementation of the AODV routing protocol in the qualnet simulator has been modified and fuzzy logic control has been embedded so as to efficiently execute the proposed algorithm.

The simulation parameters that have been used in our experiments are stated in table III.

Table III. Simulation Parameter

$\begin{array}{ll}\text { Parameter } & \text { Value } \\ \text { Transmitter range } & 250 \\ \text { Bandwidth } & 2 \mathrm{Mbps} \\ \text { Interface queue length } & 50 \text { messages } \\ \text { Simulation time } & 900 \text { seconds } \\ \text { Pause time } & 0 \text { second } \\ \text { Packet size } & 512 \text { bytes } \\ \text { Topology size } & (1000 * 1000) \mathrm{m}^{2} \\ \text { Number of Nodes } & 25,50,75 \text { and } 100 \text { nodes } \\ \text { Data traffic } & \text { CBR } \\ \text { Mobility model } & \text { Random waypoint }\end{array}$

Extensive simulation experiments have been conducted to compare the performance of AODV, distance aware counter based broadcast [DACBB] and the fuzzy logic based Probabilistic-AODV [FPBB] and [15]

\section{RESUlt AND ANALYSIS}

The analysis and comparison is done by considering two different settings, each designed to assess the impact of a particular network condition on the performance of the protocol.

Firstly, the impact of network density or size is assessed by deploying 25, 50, 75 and 100 mobile nodes over a fixed network size of 1000 by 1000 square meters. The second setting investigates the effects of offered traffic load on the performance of the routing protocols by varying the number of source destination pairs over the range of 5, 10, 15, 20 and 25 flows for each simulation scenario.

Figure 6, 7 and 8 shows the performance of AODV, DACBB and FPBB in terms of network throughput, saved rebroadcast and reachability versus network density. As shown, in the figures, the network throughput, saved rebroadcast and reachability generated by FPBB increases almost linearly as the network density increases. 


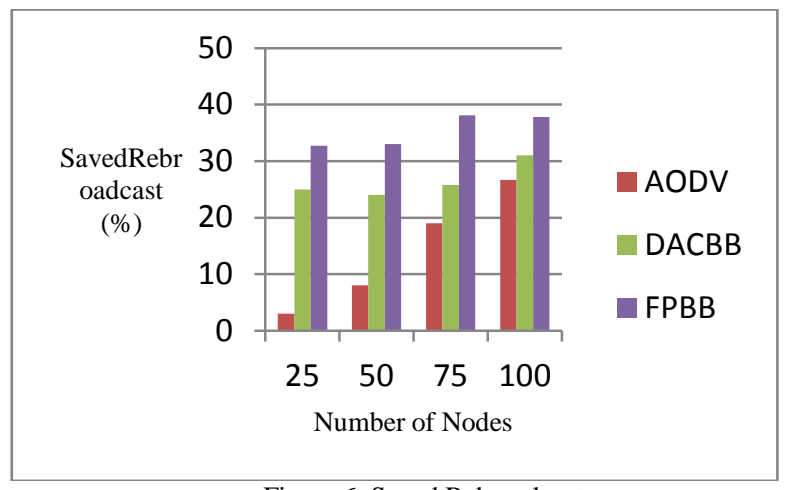

Figure 6. Saved Rebroadcast

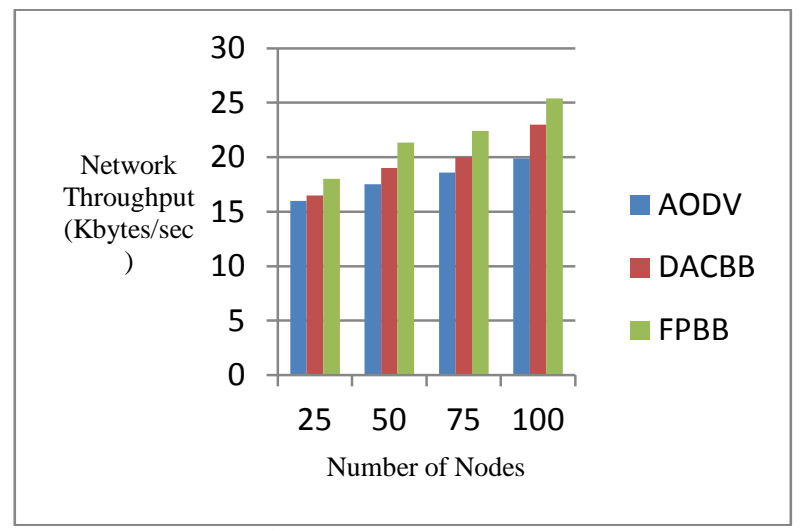

Figure 7. Network Throughput

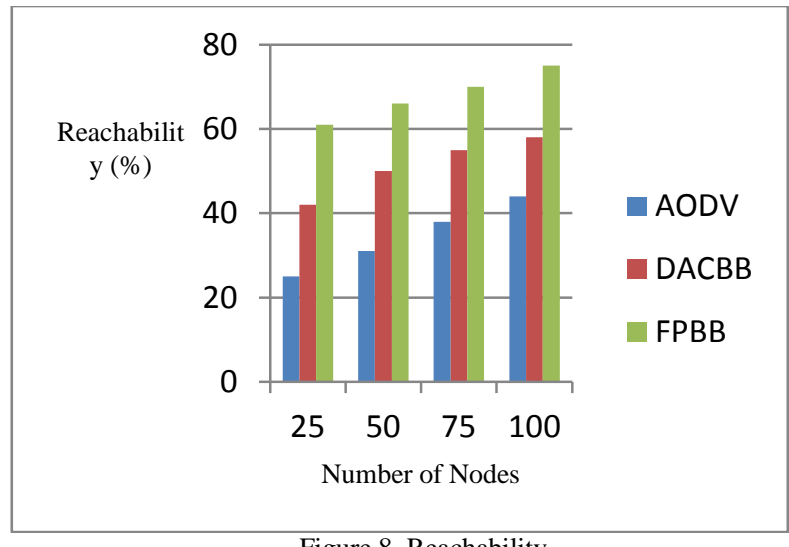

Figure 8 . Reachability

Network throughput for AODV, distance aware counter based broadcast [DACBB] and the fuzzy logic based Probabilistic-AODV [FPBB] are similar and increases almost linear when the offered load is increased from 5 to 15 flows. This is because when the number of flows is increased, the number of nodes initiating route discovery operation is also increased. As a consequence, more RREQ packets are generated and transmitted which leads to a high consumption of the communication bandwidth. This phenomenon leads to a fewer number of data packets delivered at the destinations, there by degrading the network throughput.

Figure 9 shows the superiority of the FPBB over the traditional AODV and DACBB becomes more noticeable in the case of high offered load (e.g. 25 flows). At offered load of 25 flows, the network throughput is increased by around 9.24 and 5.09 percent respectively, when compared against the
AODV and DACBB. The difference in the achieved network throughput is due to the reduction of the number of nodes involved in the dissemination of RREQ packets in congested networks, leading to a reduction of routing overhead and packet collisions. As a consequence more communication bandwidth is freed for data transmission.

The FBPP has the least number of rebroadcast for almost all traffic loads. We vary the traffic load by using different number of CBR source-destination connections. Figure 10 shows that as the number of connection increases the rebroadcast is saved more. Whereas when the number of CBR connection increases figure 11 shows that reachability also increases linearly with the increase in traffic load.
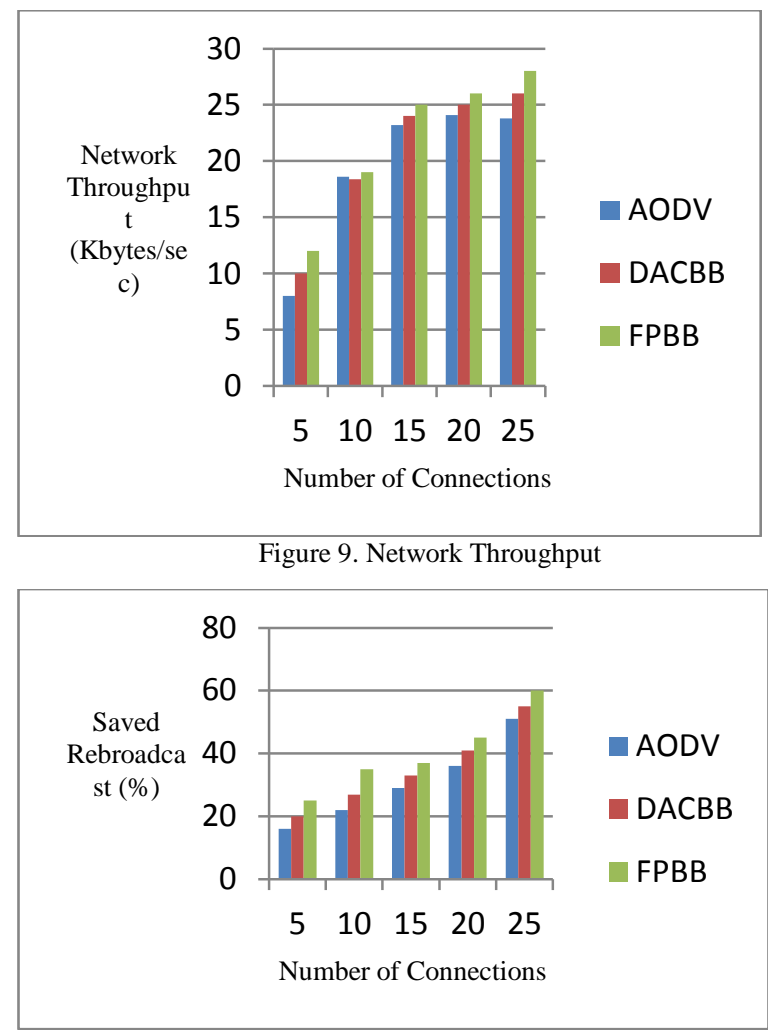

Figure 10. Saved Rebroadcast

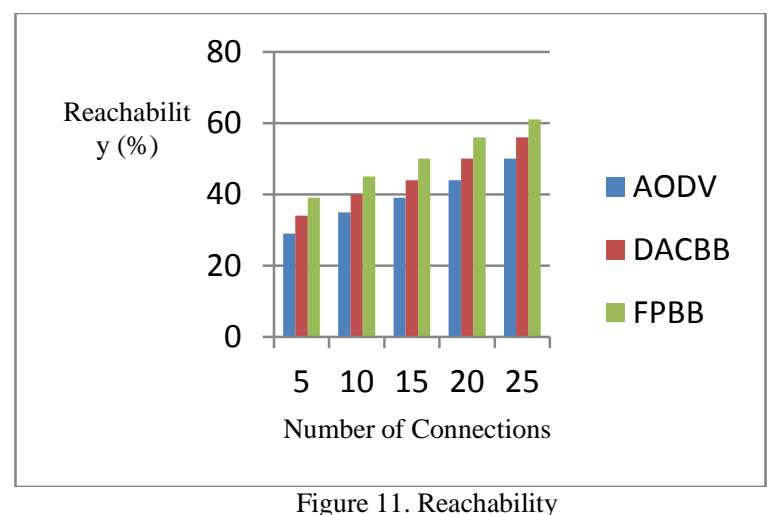

Simulation results reveal that FBPP technique outperforms DACBB in most considered performance metrics such as saved rebroadcast and reachability, while maintaining comparable performance in other important performance 
characteristics of the network such as throughput, reachability and saved rebroadcast.

\section{CONCLUSION AND FUTURE SCOPE}

This paper has evaluated the performance of fuzzy logic based distance broadcasting scheme with distance aware counter based broadcast. The present work brings out the potential advantages of applying Fuzzy Logic Control technique for generating dynamic probability value based on the node location. Fuzzy Logic Control can therefore be an effective strategy for generating varying probability value for broadcasting in MANET.

The simulation results revealed that the proposed algorithm generates much higher throughput and saved rebroadcast. The results have also shown that the degradation of the number of RREQ packet initiated and forwarded in dense network has significantly reduced. Though the analysis in this paper has been very crude, but this clearly depicts the advantage of adding the fuzzy logic controller in the conventional probabilistic broadcasting scheme. The results tend to be more broadcasting efficient. The comparisons show the superiority of Fuzzy Logic Control scheme over the smart probabilistic broadcasting schemes. As a continuation of this research work in near future, we plan to further explore the performance of the fuzzy logic based Probabilistic-AODV [FPBB] using on-demand routing protocols such as DSR. The fuzzy logic based Probabilistic-AODV [FPBB] can also be used to examine the effect on the routing table advertisements in proactive routing protocols, such as OLSR, and ZRP.

We can further explore the fuzzy logic based ProbabilisticAODV [FPBB] with varying mobility model. In the present work random waypoint mobility model is used, other model such as community based mobility model, gauss mobility model, manhattan mobility model and others can be used to study the effect on the performance of the algorithm.

\section{REFERENCES}

[1] B. Williams and T. Camp, "Comparison of broadcasting techniques for mobile ad hoc networks," in Proceedings of the 3rd ACM international symposium on Mobile ad hoc networking. ACM Press, 2002, pp. 194205.

[2] W. Lou and J. Wu, Localized Broadcasting in Mobile Ad Hoc Networks Using Neighbor Designation. CRC Press, 2003.

[3] F. Dai and J. Wu, "Distributed dominant pruning in ad hoc networks," in ICC, 2003.

[4] Ho, C., K. Obraczka, G. Tsudik and K. Viswanath, 1999. Flooding for reliable multicast in multi-hop ad hoc networks. Proceedings of the International Workshop on Discrete Algorithms and Methods for Mobile Computing and Communication, Aug. 20- 20, Seattle, Washington, United States, pp: 64-71. http://portal.acm.org/citation.cfm?id=313291

[5] Jetcheva, J., Y. Hu, D. Maltz and D. Johnson, 2001. A simple protocol for multicast and broadcast in mobile ad hoc networks. Internet Draft: draft-ietf-manet-simple-mbcast-01.txt.

[6] Ni, S., Y. Tseng, Y. Chen and J. Sheu, 1999. The broadcast storm problem in a mobile ad hoc network. Proceedings of the ACM/IEEE International Conference on Mobile Computing and Networking, Aug. 15-19, Seattle, Washington, United States, pp: 151-162. http://portal.acm.org/citation.cfm?id=313451.313525.
[7] Sucec, J. and I. Marsic, 2000. An efficient distributed network-wide broadcast algorithm for mobile ad hoc networks. CAIP Technical Report 248-Rutgers University. http://citeseer.ist.psu.edu/312658.html

[8] Y.-C. Tseng, S.-Y. Ni, and E.-Y. Shih, "Adaptive approaches to relieving broadcast storms in a wireless multihop mobile ad hoc networks," Proceedings of IEEE Transactions on Computers, vol. 52, pp. 545-557, May 2003.

[9] Q. Zhang and D. P. Agrawal, "Dynamic probabilistic broadcasting in MANETs," Journal of Parallel and Distributed Computing, vol. 65, pp. 220-233, 2005.

[10] M. B. Yassein, M. O. Khaoua, L. M. Mackenzie, and S. Papanastasiou, "The Highly Adjusted Probabilistic Broadcasting in Mobile Ad hoc Networks," Proceedings of the 6th Annual PostGraduate Symposium on the Convergence of Telecommunications, Networking \& Broadcasting, (PGNE T 2005), vol. ISBN 1- 902-56011-6, pp. 27-28, June 2005.

[11] Y. Sasson, D. Cavin, and A. Schiper, "Probabilistic broadcast for flooding in wireless mobile ad hoc networks," Proceedings of IEEE Wireless Communications and Networking Conference (WCNC), March 2003.

[12] W. Peng and X. C. Lu, "On the reduction of broadcast redundancy in mobile ad hoc networks," proceedings of the ACM Symposium on Mobile and Ad Hoc Networking and Computing (MobiHoc'00), pp. 129130, August, 2000

[13] J. Wu and W. Lou, "Forward-node-set-based broadcast in clustered mobile ad hoc networks," Wireless Communications and Mobile Computing, vol. 3, pp. 155- 173, March 2003.

[14] C. Perkins, E. Belding-Royer, and S. Das, "Ad hoc On-Demand Distance Vector (AODV) Routing," IETF Mobile Ad Hoc Networking Working Group INTERNET DRAFT, RFC 3561,July 2003, http://www.ietf.org/rfc/rfc3561.txt. Experimental RFC, retrieved in October 2007.

[15] Chien Chen, Chin-Kai Hsu, and Hsien-Kang Wang," A distance-aware counter-based broadcast scheme for wireless ad hoc networks",Military communication conference-2005 IEEE, 17-20 oct, Pages 1052-1058, Vol-2.

[16] L.Zhou, G.Cui, H.Liu, Wu and D.Luo "NPPB: A Broadcast scheme in Dense VANETs", Information Technology Journal 9 (2): 247-256, 2010, ISSN 1812-5638, 2010 Asian Network for Scientific Information.

[17] Tasneem Bano, Jyoti Singhai "Probabilistic broadcasting Protocol in ad hoc network and its advancement: a Review", IJCSES volume 1, number 2, Nov 2010, AIRCC publicationISSN-0976-2760 Online ISSN-09763252 Print.

\section{AUTHORS PROFILE}

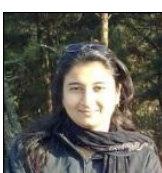

Tasneem Bano Rehman is a research scholar in Computer Science engineering department in Maulana Azad National Institute of Technology (MANIT). She holds a Master of Technology and Maulana Azad National Institute Of Technology. Her general research interests include wireless communication especially Mobile Ad-hoc Networks.

Dr. Jyoti Singhai is Associate Professor in Electronics

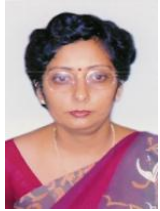
and Communication Engineering Department in Maulana Azad National Institute of Technology (MANIT) since 1994. She did her BE in Electronics Engineering in 1991 from MANIT (formerly known as Maulana Azad College of Technology), Bhopal. She did her M.Tech. in Digital Communication in 1997 and $\mathrm{PhD}$ in 2005 from MANIT, Bhopal. She is recipient of "Young Scientist Award" from M.P Council of Science and Technology, Government of M.P. for the year 2002-03, "Career Award for Young Teachers" form AICTE in 2008 and "BOYSCAST Fellowship" from DST in 2010. She has published over 80 papers in various National and International conferences. She has supervised 40 UG Major projects, 41 M.Tech. projects and $6 \mathrm{PhD}$. 